\title{
Research on the Simulation of Face Terminal Identification under the Network Security of Mobile Devices
}

\author{
Zhao Xianghui \\ School of Software and Big Data, Changzhou College of Information Technology, Changzhou, Jiangsu, \\ 213164, China \\ email:27898063@qq.com
}

Keywords: Mobile Device, Face Terminal, Identity Recognition

\begin{abstract}
The face terminal identity recognition under the network security of mobile terminal can effectively guarantee the security of user identity information. In order to carry out face terminal authentication, it is necessary to allocate memory resources to the face terminal device and guide it. It is necessary to mark the best solution set of the face to complete recognition. The existing methods are face feature image collection, these detection, tracking, and recognition results in order to get the guard terminal database information and consistent face, the annotation of the best scene of the face ignores the recognition accuracy, as a result. A face terminal recognition method based on face detection and recognition is proposed. In order to improve the use of AdaBoost method detection, the direction of face, and determine the candidate domain division based on the exploration information of local features based on skin color features and the difference quality of nearest neighbor classification method, the image flow of face terminal, the cognitive ability of face improvement, and the implementation guide of memory resource allocation for the best solution set of annotation. In addition, in order to realize the face terminal recognition of mobile device network security, a sample set placed in the memory of mobile device is selected. The experimental results show that the proposed authentication method can fully realize the recognition of mobile devices.
\end{abstract}

\section{Introduction}

In recent years, with the popularization and development of computer technology and mobile devices, such as smart phones, the effective and convenient network security of mobile devices has been paid more and more attention [1]. The identification technology based on bioassay has the security advantages of being difficult to use maliciously and improperly, forgery and so on. With the rapid development of $3 \mathrm{G}$ network and the continuous strengthening of mobile device hardware configuration, it is possible to develop the face terminal recognition technology. With the rapid development of computer technology and the popularization of mobile devices such as smart phones, people pay more and more attention to the network security of mobile devices. As mentioned in the literature, face recognition technology has attracted the attention of the industry in the academic field due to its very good security. As a biometric recognition technology, there are some difficult features such as bullying and forging. As a research hotspot in the field of pattern recognition, face recognition is more efficient and effective than biometric features such as fingerprint, retina and walking. The development of mobile 4G network and the increase of mobile device hardware provide a solid foundation for the development of mobile device terminal authentication technology. As the most efficient and fast recognition method in daily life, face recognition technology has always been a research hotspot in the field of pattern recognition. In this study, although the recognition method based on iris and fingerprint is more accurate than face recognition, the non-contact, non obligation and simultaneity characteristics of face recognition suggest that people are easy to accept. Because users do not need to contact the device directly, face recognition can obtain samples without user awareness [2]. A camera or camera connected to a computer is used to collect, detect, and track facial feature images, videos. Therefore, this paper proposes a face terminal recognition method based on face detection and recognition. Initially, skin 
color features were used to quickly exclude most of the background areas through multi pose face detection methods improved based on facial features and AdaBoost theory. Then, the direction of face is that candidates use the AdaBoost theory to prepare for the field of classification to divide the candidate field into different local search features that are easy to flesh color field, so they decide to improve the performance of face recognition. In order to improve the quality of the classified recognition information and the highest position, after the sample disk, they select the best subset of faces, and the memory of the recognition mobile device is memorized, The final face recognition is based on the recognition of the completion port.

\section{The Principle of Face Terminal Identity Recognition under the Network Security of Mobile Devices}

In order to use the face terminal to authenticate the identity, the skin color of the face needs to be detected first [3]. The skin color space of the face is only a small part of the whole chroma space. Clustering is very strong. This paper points out that the facial image detector is made according to the facial features such as skin color and AdaBoost theory, and the image information is analyzed and classified. The detection of skin color completes the complex background from the field of skin color. In order to separate the skin color using function classification, eye, mouth and skin color on the YCbCr color space, we will search other areas of the field, the direction of the face, the geometric characteristics of the obtained, there are also candidate face areas, the final candidate face, AdaBoost classification theory and complete. The color difference of people with different skin color is very small, and the difference of brightness is the main difference of skin color. Each component has content information in RGB color space, has strong correlation and is easy to be affected by light. In order to reduce the influence of illumination change, the YC BC r color space is transformed into yc'bc'r color space by segmented nonlinear color space transformation. In YC 'BC' $\mathrm{R}$ nonlinear color space, when the pixel satisfies the equation, it can be considered as skin color pixel [4]. Otherwise, because it is not a skin color pixel, binary image can be easily obtained. Through morphological processing, the skin color area can be detected correctly. Through the geometric characteristics of the skin color wiring area, the obvious non facial area can be completed, and the obvious non facial area can be removed. In order to get a general face recognition region, we need to search the eyes, mouth and other regions first, and then use the geometric function to complete the face direction recognition. The face candidate area is segmented in that direction. The components of c'b and c'of eyes and mouth are quite different in the color space of yc'bc'r. The c'b component of eyes is higher than that of c'r. By combining with luminance mapping, the size and position of eye area can be accurately detected. The mouth is an obvious non skin area in facial features. The $\mathrm{C}$ component of mouth color is higher than the c'b component, and the difference between the two is larger. It can detect the size and location of the port area. The determination of the face direction is performed by using the attachment angle between different regions. Then, the corresponding face candidate region can be segmented. Then, the cascaded AdaBoost detection classifier is used to complete the face candidate region classification.

\section{Face Terminal Identification Method}

The face recognition system performs the face recognition process through the nearest neighbor segmentation model. After this operation, the classified face image is displayed to the user, and the user selects the corresponding face label for correction [5]. Maximize the combination of user feedback and the updated neighborhood model. In this article, the most precious samples for classification and recognition are only samples, but the mobile device recognition process is memorized for better implementation of streaming data sampling. Human face samples. There is only one memory B in the face terminal device. Example of maxcount buffer. For a period of time, the system searches the data record closest to the memory for each initial acquired sample Xi to match the new label of the updated sample. After performing the classification, the new label YT is updated in memory. For the limited storage space of mobile devices, face recognition problem can 
be transformed into selecting the best face data subset, including the most valuable samples. In this paper, it is necessary to quantify the value of each sample to prepare for a good classification later[6]. The quantized value is used as the best criterion for memory selection. In the actual recognition process, it is necessary to use the sample of probability distribution to solve the expected value of information (EVI). Due to the uneven characteristics of different examples, the ability of difference recognition is also different. In order to estimate the differential recognition performance of each sample in the memory, several test samples are considered. The corresponding distance between the test sample point and the subset point in the memory is used to determine the sample value of the sample. This system is mainly to keep the sample of memory buffer, so the classifier can get the maximum standard value. The sample points to be measured can be realized backward through classification, and can be completed according to the recognition processing of face recognition terminal device.

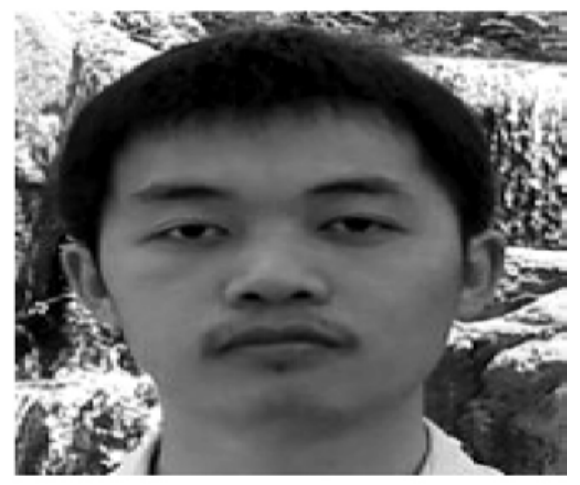

Figure 1 Original image of facial eye feature recognition

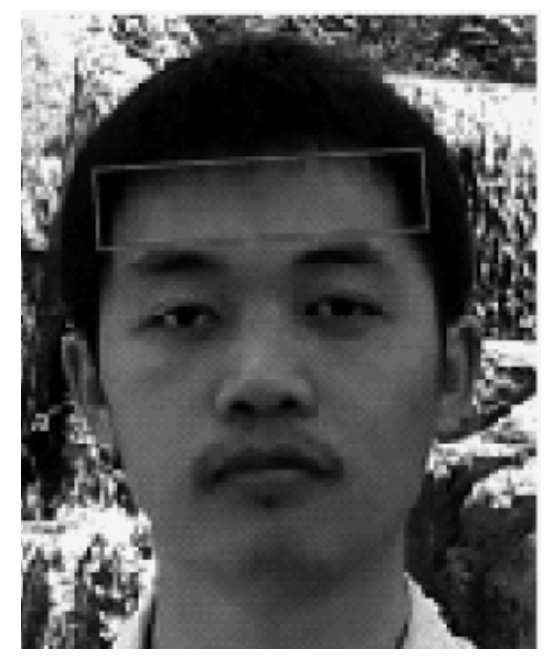

Figure 2 Recognition results of local feature method

\section{Experiment and Result Analysis}

The bioassay is unique and characteristic. In order to verify the feasibility of face terminal recognition based on face recognition, the following experiments are carried out[7]. Three facial feature images are given in the experiment. The text cascade structure of AdaBoost face detection classifier and local feature analysis method are used for face recognition in the above three images. Save the recognition process. After this operation, the classified face image is displayed to the user, and the user selects the corresponding face label for correction. Maximize the combination of user feedback and the updated neighborhood model. In this paper, only the most valuable samples for classification and identification are stored when the data is sampled for streaming transmission, so as to better perform the mobile device identification process. Human face samples $\{\mathrm{x} 1$, $\mathrm{x} 2$ Set the system for storing, XR2\}. There is only one memory B in the face terminal device. Example of maxcount buffer. For a period of time, the system searches the data record closest to the memory 
for each initial acquired sample Xi to match the new label of the updated sample. After performing the classification, the new label YT is updated in memory. For the limited storage space of mobile devices, face recognition problem can be transformed into selecting the best face data subset, including the most valuable samples. In this paper, it is necessary to quantify the value of each sample to prepare for a good classification later. The quantized value is used as the best criterion for memory selection. In the actual recognition process, it is necessary to use the sample of probability distribution to solve the expected value of information (EVI). Due to the uneven characteristics of different examples, the ability of difference recognition is also different [8]. In order to estimate the differential recognition performance of each sample in the memory, several test samples are considered. The corresponding distance between the test sample point and the subset point in the memory is used to determine the sample value of the sample. This system is mainly to keep the sample of memory buffer, so the classifier can get the maximum standard value. The sample points to be measured can be realized backward through classification, and can be completed according to the recognition processing of face recognition terminal device. Recognition is to compare the differences between two facial features, such as the recognition of eyes, nose and mouth. The above image is the original image of eye feature recognition. The AdaBoost face detection classifier with cascaded structure in text is used to classify and recognize the facial features of images. The middle red field is the result of text recognition. Compare images using local feature methods. According to the results of recognition analysis, the method in this paper can recognize the facial features of eyes more accurately. In order to recognize the original image of face and nose features and the original image of face mouth function, the two images are also analyzed, and the AdaBoost face discovery classifier with text cascade structure is used for processing. In the image, the facial features, nose features and mouth features can be recognized correctly [9]. After using the local feature method to classify the face detection image, the image can be observed clearly. It can be seen that the recognized nose feature area and mouth feature area are not correct, and can not correctly identify the purpose of the facial feature area. After the implementation of the feature face recognition experiment, the information authentication method predicts the used value, sample and sample, and uses the face and sample verification device to verify the integrity of the system. In order to implement the peak of the test classification sample, the terminal authentication and key distribution face methods are compared, and the classification accuracy of the test sample data is analyzed and compared. Before face terminal authentication, face image data must be classified correctly. By using the expected value method and key distribution method of the above four data groups to classify the sample data to be tested, the key distribution method is used. The classification accuracy of the test data is less than $50 \%$, which means that it can not meet the authentication requirements of the face terminal device using a large amount of information. The accuracy of the sample data classification using the expected value method of information in the text reaches 99\%. The method shown in the text provides stable data support for authentication.

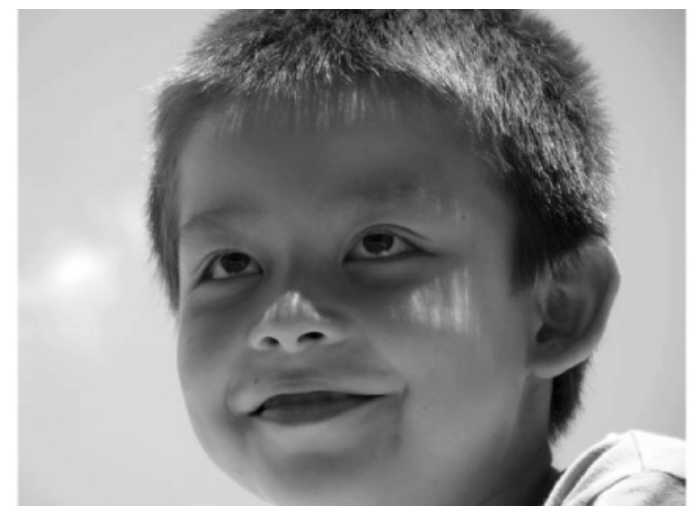

Figure 3 Original image of face mouth feature recognition 


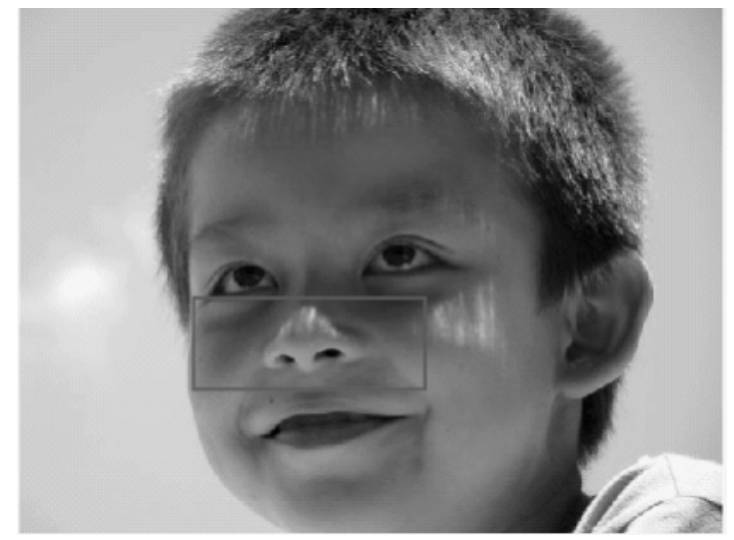

Figure 4 Recognition results of mouth feature area by local feature method

\section{Conclusion}

In order to enhance the security performance of wireless mobile devices, a face terminal authentication method for network security of mobile devices is proposed[10]. First of all, we use the improved facial detection method based on facial features and AdaBoost theory to explore the local facial features of the region after using the skin background features in order to exclude the background, and correctly segment the candidate region. Secondly, according to the AdaBoost theory, the candidate feature areas are classified, the best facial solution set is annotated, and the sample set placed in the mobile device memory is selected to realize the face terminal recognition of mobile device network security. The experimental results show that the proposed authentication method can fully realize the recognition of mobile devices.

\section{Acknowledgements}

This research has been supported by Natural Science Project of Changzhou College of Information Technology “Face Recognition from Deep Features”2018 (CXZK201803Z).

\section{References}

[1] Alison Campbell, James W. Tanaka. Inversion Impairs Expert Budgerigar Identity Recognition: A Face-Like Effect for a Nonface Object of Expertise. Perception, vol. 47, no. 6, pp. 030100661877180, 2018.

[2] Zhang J, Qiang L, Wang X, et al. Towards fast and lightweight spam account detection in mobile social networks through fog computing, no. 4, pp. 1-15, 2017.

[3] Augusto A B. OFELIA - A Secure Mobile Attribute Aggregation Infrastructure for UserCentric Identity Management, vol. 376, 2017.

[4] Kelly C, Spencer S, Grundy S, et al. Self-management for non-cystic fibrosis bronchiectasis: Cochrane Systematic Review, vol. 2017, no. 1, pp. A114.2-A115, 2017.

[5] Elena Panagiotopoulou, Maria Laura Filippetti, Manos Tsakiris,. Affective Touch Enhances Self-Face Recognition During Multisensory Integration. Scientific Reports, vol. 7, no. 1, 2017.

[6] Tang Wenjing, Gong Fei, Dong Renren,. Face recognition based on the fusion of wavelet packet sub-images and fisher linear discriminant. Multimedia Tools \& Applications, vol. 76, no. 21, 2017.

[7] Marcin Masalski, Tomasz Grysiński, Tomasz Kręcicki. Hearing Tests Based on Biologically Calibrated Mobile Devices: Comparison With Pure-Tone Audiometry. Jmir Mhealth Uhealth, vol. 6, no. 1, pp. e10, 2018.

[8] Gayathri Mahalingam, Karl Ricanek, A. Midori Albert. Investigating the Periocular-Based Face Recognition Across Gender Transformation. IEEE Transactions on Information Forensics \& 
Security, vol. 9, no. 12, pp. 2180-2192, 2017.

[9] Anne Oeldorf-Hirsch, Jeremy Birnholtz, Jeffrey T. Hancock. Your Post is Embarrassing Me:

Face Threats, Identity, and the Audience on Facebook. Computers in Human Behavior, vol. 73, pp. 92-99, 2017.

[10] Hao ZHOU, Hong-feng CHAI, Mao-lin QIU. Fraud detection within bankcard enrollment on mobile device based payment using machine learning, 2018. 